\title{
EHD TURBULENCE IN CHANNEL FLOWS WITH INHOMOGENEOUS ELECTRIC FIELDS: A ONE-DIMENSIONAL TURBULENCE STUDY
}

\author{
HEIKO SCHMIDT $^{1}$, JUAN A. MEDINA MENDEZ ${ }^{1}$ AND MARTEN KLEIN ${ }^{1 *}$ \\ ${ }^{1}$ Numerical Fluid and Gas Dynamics, Brandenburg University of Technology Cottbus-Senftenberg \\ Siemens-Halske-Ring 15A, D-03046 Cottbus, Germany \\ *Contact: marten.klein@b-tu.de • https://www.b-tu.de/en/fg-stroemungsmodellierung/
}

Key words: Stochastic modeling, one-dimensional turbulence, channel flow, EHD turbulence

\begin{abstract}
Electricly enhanced flows can be found in various technical applications as, for example, in air cleaning devices and liquid metal or redox flow batteries. For both examples mentioned it is crucial to develop a general understanding of the relevant physical processes and model them economically. Additionally, a correct upscaling procedure is specifically relevant for the transition into the industrial scale. All of these aspects are challenging because of the multiscale and multiphysics nature of these flows. In this paper we present a lower-order modeling strategy that aims to bridge the gap between fundamental research and applications by utilizing stochastic one-dimensional turbulence (ODT). Two case studies are performed. One is for two-way coupled turbulent Couette flow of electrolytes and another for one-way coupled planar Poiseuille flow in a wire-plate electrostatic precipitator. By comparison with reference data, we show that the modeling approach is robust and has predictive capabilities. Nevertheless, we also discuss some limitations of the purely one-dimensional and stochastic dynamical representation.
\end{abstract}

\section{INTRODUCTION}

We introduce some aspects of the nature of turbulence by categorizing the research and applications of electrohydrodynamic (EHD) flows as follows. In a first category, EHD effects are subordinated to the flow dynamics and do not play a relevant role, except for the enhancement of specific properties of an application, as in EHD-enhanced heat exchangers [1,2] or plasma-assisted flames [3]. In a second category, EHD effects determine the nature of the application and, thus, have leading order effects on the flow, as in the case of industrial electrostatic precipitation [4, 5] or flow control [6]. The electrokinetics and the flow are affected by transient processes on all relevant scales, such that robust, accurate, but also economical modeling strategies, are required to tackle these flows. This is addressed here by utilizing the stochastic one-dimensional turbulence (ODT) model [7, 8], recently extended to incorporate EHD effects by a one-way [9], and a two-way [10] coupling strategy, respectively.

We apply ODT to two cases from the topics of electrostatic precipitation and turbulent flow control. For that, we distinguish a limiting 'adiabatic' regime (electric fields in equilibrium) and a fully coupled regime. For the 'adiabatic' regime, we consider a planar precipitator, which is given by a channel with wire electrodes in the mid plane. These electrodes yield a spatially nonhomogeneous background electric field that couples to the free charges in the working fluid. The fluid does not have any feedback effect on the background electric field, that is, a one-way coupling as in [5]. For the fully-coupled regime, we consider a planar channel with moving walls held at different voltages. The working fluid possesses 
positive and negative ion species so that charge separation enables electric drift currents that may interact with the flow. Our results exhibit good to reasonable agreement with available references data for the mean and low-order fluctuation statistics, respectively. Furthermore, the results signalize the importance to capture the spatio-temporal structure of the electric field.

The paper is organized as follows. In section 2 we describe the model formulation and its application to EHD turbulence. In section 3 we present and discuss ODT results for the two-way (fully) coupled case of turbulent EHD Couette flows of electrolytes. In section 4 we present and discuss ODT results for the one-way coupled case of the electricly-enhanced turbulence in planar Poiseuille flow. In section 5 we state some concluding remarks.

\section{OVERVIEW OF THE MODEL FORMULATION}

Kerstein's [7] one-dimensional turbulence model aims to resolve all relevant scales of a turbulent flow by a dimensional model reduction. The effects of Navier-Stokes turbulence are modeled by a stochastic process acting on a notional line-of-sight. The computational domain is, hence, one-dimensional and denoted as the ODT line. In the following, some aspects of the model formulation are discussed, which are relevant for the model application to EHD turbulence.

\subsection{ODT governing equations for momentum conservation in EHD flows}

We consider constant-property EHD flows of weakly conducting fluids that are described by the NavierStokes equations coupled to the Poisson-Nernst-Planck equations and an equation for the electric potential (see, for exampl field is prescribed so that diluted. The more general charge carrier densities background electric field.
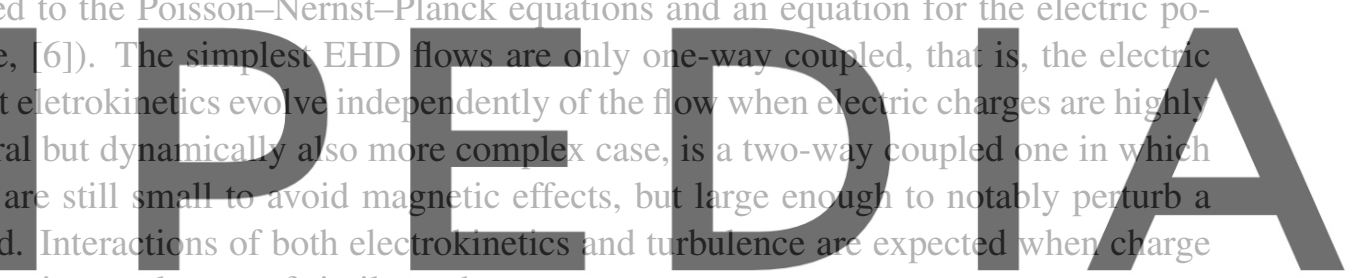

relaxation and convective time scales are of similar order.

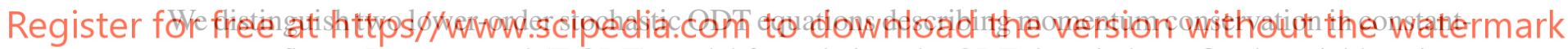
property flows. In a temporal (T-ODT) model formulation, the ODT domain has a fixed spatial location and aims to resolve the temporal evolution of flow properties along the resolved direction. The lowerorder momentum equation for incompressible flow is a 1-D partial differential equation (PDE),

$$
\frac{\partial \vec{u}}{\partial t}+\mathcal{E}_{\vec{u}}=\frac{\partial}{\partial y}\left(v \frac{\partial \vec{u}}{\partial y}\right)-\vec{e}_{x} \frac{1}{\rho} \frac{\mathrm{d} P}{\mathrm{~d} x}
$$

where $y$ denotes the wall-normal (ODT line) coordinate, $t$ the time, $\vec{u}=(u, v, w)^{\mathrm{T}}$ the velocity vector with its conventional Cartesian components in streamwise, wall-normal, and spanwise direction, respectively, $\rho$ and $v$ the fluid's constant mass density and kinematic viscosity, respectively, $\vec{e}_{x}(\mathrm{~d} P / \mathrm{d} x)$ a streamwise mean pressure gradient acting as a momentum source (set to zero for Couette flow). $\mathcal{E}_{\vec{u}}$ represents the effects of turbulent eddies for the velocity vector and is formulated by discrete stochastic mappings, denoted as 'eddy events' that are detailed below. We only note here that $\mathcal{E}_{\vec{u}}=0$ between two subsequent eddy events which yields piecewise continuous advancement of deterministic molecular processes [7] like diffusion.

In a spatial (S-ODT) formulation, the ODT domain itself is not stationary but advected by the flow in 
streamwise direction in the course of a simulation run. This formulation assumes a statistically stationary 2-D turbulent field that will have a parabolic character in the model solution and is determined by initial conditions, here to be taken as inflow. For S-ODT, the temporal rate of change of $\vec{u}$ in equation (1) is replaced by a streamwise advection term that yields evolution along the streamwise coordinate $x$ instead of time $t$ which yields

$$
u \frac{\partial \vec{u}}{\partial x}+\mathcal{E}_{\vec{u}}=\frac{\partial}{\partial y}\left(v \frac{\partial \vec{u}}{\partial y}\right)-\vec{e}_{x} \frac{1}{\rho} \frac{\mathrm{d} P}{\mathrm{~d} x}
$$

For EHD enhanced flows, we need to furthermore consider the Poisson-Nernst-Planck equations and an equation for the electric potential. Due to the distinctive nature of the application cases evaluated in this study, the latter referenced equations are presented below, in the context of each application case.

The deterministic parts of (1) and (2) are integrated in the context of a time or streamwise marching scheme, respectively. The spatial discretization along the ODT domain (wall-normal coordinate $y$ ) is performed with a Lagrangian finite volume scheme on an adaptive grid as described in [11].

\subsection{Overview of ODT eddy events and model parameters in EHD turbulence}

This section provides an overview of some relevant aspects of the ODT eddy event formulation but does not aim to be complete. For technical details we defer the reader to the literature cited.

In equation (1) and (2), $\mathcal{E}_{\vec{u}}$ represents the effects of turbulent advection as well as fluctuating pressure-

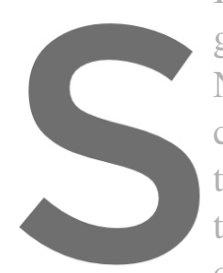

gradient and Coulomb forct

Nernst-Planck equation

corporate the effects of tur

term that affects the scala

tinguish velocity $\left(\mathcal{E}_{\vec{u}}\right)$ fron

conversion has been introduced by
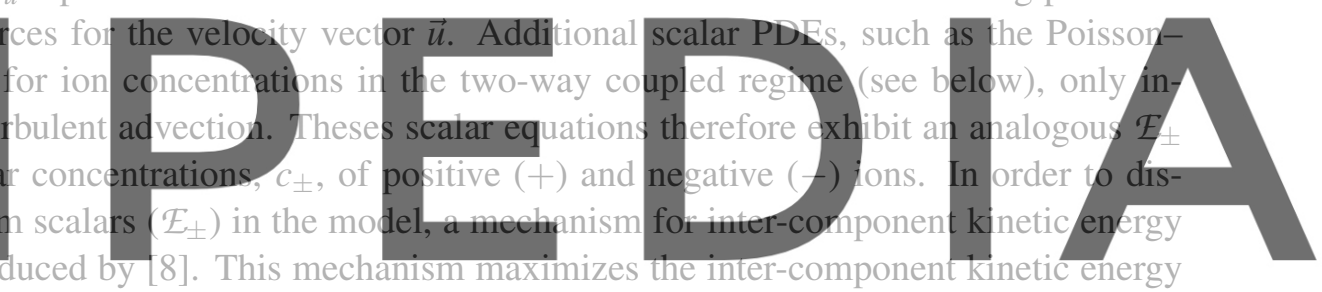

exchange due to pressure-velocity couplings by a model parameter $\alpha$ that affects $\mathcal{E}_{\vec{u}}$ but not $\mathcal{E}_{ \pm}$. For

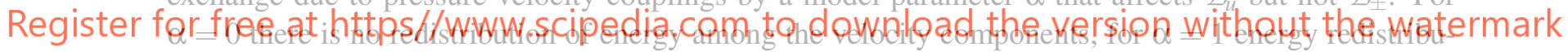

tion is maximized, and for $\alpha=2 / 3$ equipartition is achieved, which maximizes the rate at which a return

to smali-scale isotropy is observed. In addition to kinetic also potential energy changes have to be considered by accounting for the action of Coulomb analogous to buoyancy forces [12]. Here we specifically use the velocity vector formulation with potential energy treatment as in [13], which has been added to the adaptive ODT implementation from [11].

In the following we specialize to the T-ODT context. ODT eddy events are implemented as a stochastic sequence of mapping events that instantaneously manipulate property profiles of $\vec{u}(y)$ and $c_{ \pm}(y)$ by application of the triplet map $f(y)$ [7] and, in the case of the velocity and/or an active scalar, by kernel $K(y)=y-f(y)$ applications [8,12]. The triplet map models the microstructure of turbulent eddies by bringing fluid from location $f(y)$ to the mapped location $y$. The operation is measure preserving and continuous, but introduces artificial discontinuous derivatives that are smoothed by molecular diffusion. In this procedure, every eddy event is characterized by two random variables, eddy location $y_{0}$ and size $l$, that, for a fixed point in time $t$, are sampled from an unknown probability density function (PDF) dependent on the current state of the flow [7]. A costly construction of this PDF is avoided in practice by using a more efficient thinning-and-rejection algorithm in which the eddy turnover time $\tau=\tau\left(y_{0}, l ; t\right)$ is 
used for the probabilistic sampling of eddy events. Correspondingly, the momentary eddy rate is

$$
\tau^{-1}=C \sqrt{\frac{2}{\rho V l^{2}}\left(E_{\mathrm{kin}}+E_{\mathrm{pot}}-Z E_{\mathrm{vp}}\right)},
$$

where $V$ is the notional eddy volume that is associated with $l, \rho$ the fluid mass density, $E_{\mathrm{kin}} \sim \sum_{i=1}^{3} u_{K, i}^{2} / 2$ the shear-extractable kinetic energy due to mapping-kernel $K(y)$ weighted velocity components $u_{K, i}=$ $\int u_{i} K \mathrm{~d} y, E_{\mathrm{pot}} \sim \int\left(\left[\rho_{\mathrm{f}} \Phi\right]_{\text {before }}-\left[\rho_{\mathrm{f}} \Phi\right]_{\mathrm{after}}\right) \mathrm{d} y$ the eddy-extractable potential energy due to hypothetical eddy implementation on the electric charge density $\rho_{\mathrm{f}}=e\left(c_{+}-c_{-}\right)$that yields the electric potential $\Phi\left(\rho_{\mathrm{f}}\right)$, and $E_{\mathrm{vp}} \sim v^{2} / l^{2}$ a viscous penalty energy for the selected scale $l$ modulo constant dimensional prefactors. Physically plausible eddy events have real-valued $\tau$ and only $E_{\text {kin }}$ and, in the two-way coupled case also $E_{\text {pot }}$, are subject to redistributed by kernel application. The coefficients $C$ and $Z$ in equation (3) denote the ODT turbulence intensity and the viscous suppression parameter, respectively. $C$ controls the mean rate of eddy events, whereas $Z$ provides an energy criterion for the smallest permissible eddy size.

Note that in the S-ODT context, energy terms in equation (3) are reinterpreted as energy fluxes, while the eddy turnover time $\tau$ is superseded by a turnover length scale in streamwise direction. Either in T-ODT or S-ODT, equation (3) is evaluated for stochastic rejection (acceptance) of any candidate eddy event that has been economically obtained from guessed individual distribution functions for $l$ and $y_{0}$. Note further that, for the one-way coupled formulation, the electric potential energy formulation follows an analogous treatment to the Darrieus-Landau formulation in [14]. That is, this is the modeling of an EHD instability by an intrinsie-mechanism and not an external force, duc to a decomposition of the electrie
current density vector. Details of the formylation can be found in [9].

3 TWO-WAY COUPL Our first application is the two-way coupled Couette the transient and nonhomogeneous electric field, how it develops, and how it affects the mean velocity.

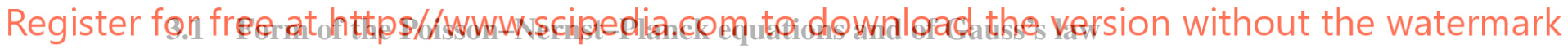

The two-way coupled case of constant-property turbulent Couette flow implements a fully one-dimensional framework. In addition to the momentum equation (1), the Poisson-Nernst-Planck equations for the ion concentrations and Gauss's law for ionic charge and electric potential, are written in a one-dimensional framework as

$$
\begin{aligned}
\frac{\partial c_{ \pm}}{\partial t}+\mathcal{E}_{ \pm} & =\frac{\partial}{\partial y}\left(D \frac{\partial c_{ \pm}}{\partial y} \pm \frac{D c_{ \pm}}{V_{T}} \frac{\partial \Phi}{\partial y}\right), \\
\frac{\partial}{\partial y}\left(-\varepsilon \frac{\partial \Phi}{\partial y}\right) & =\rho e\left(c_{+}-c_{-}\right),
\end{aligned}
$$

where $c_{ \pm}$is the positive/negative ion concentration, $D$ the diffusion coefficient of the ions, $\Phi$ the electric potential, $V_{T}=k_{\mathrm{B}} T / e$ the thermal voltage, $k_{\mathrm{B}}$ the Boltzmann constant, $T$ the temperature, $e$ the (positive) electric charge of an electron, $\varepsilon$ the dielectric permittivity of the electrolyte. $\mathcal{E}_{ \pm}$is the stochastic term representing the turbulent advection effects on the scalar ion concentration, such that $\mathcal{E}_{ \pm}$is zero between two subsequent eddy events, entirely analogous to $\mathcal{E}_{\vec{u}}$. 

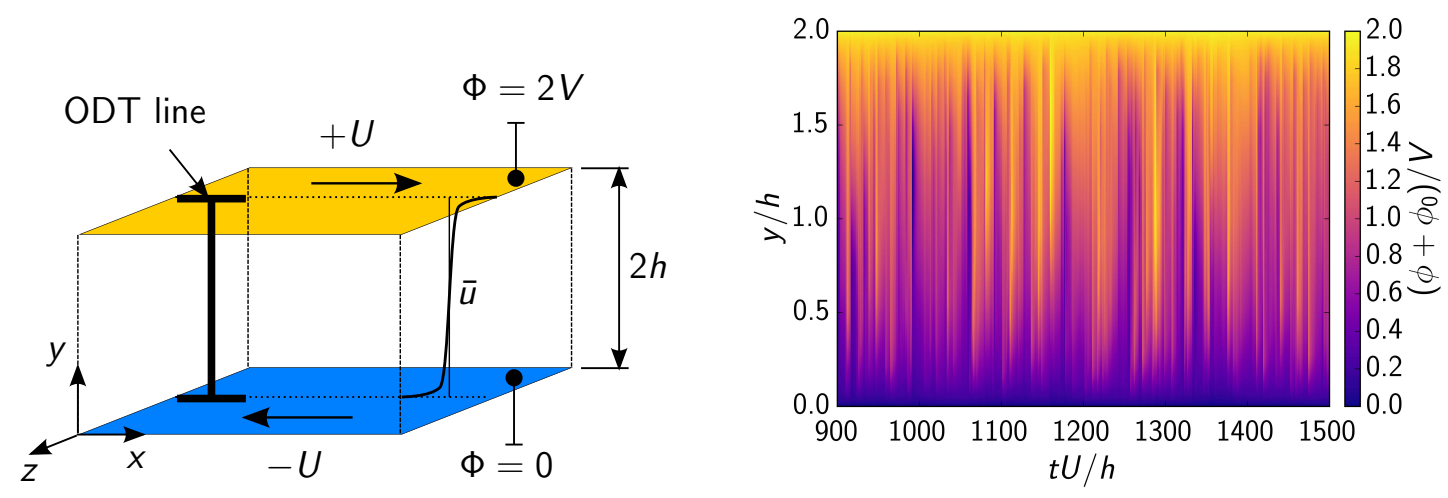

Figure 1: [left] Sketch of the EHD Couette flow configuration. The ODT domain for self-contained flow simulations is denoted as 'ODT line'. [right] ODT simulated space-time diagram of the fluctuating total electric potential $\Phi=\phi+\phi_{0}$ in turbulent electrolyte flow with for $R e=3000, S c=3, \hat{V}=4, \beta=1 / 2$, and $\hat{\lambda}_{\mathrm{D}}=0.03$.

Equation (4) is integrated with a time-marching scheme, in an entirely analogous way to equation (1). Conversely, the Thomas algorithm is used to solve equation (5) in the two-way coupled configuration.

\subsection{Flow configuration and control parameters}

Figure 1 shows a sketch of the two-way coupled flow configuration investigated. The ODT domain ('ODT line') for self-contained flow simulations is aligned with the vertical or wall-normal (y) direction and spans the entire height $2 h$ of the channel. The lateral directions $(x, z)$ are taken to infinity so that
the flow is statistically invariant in these directions. Within the stand-alone model application we do not
resolve the flow in $x$ and $z$ directly but medel the effects of Navier-Stokes turbulence by stochastic eddy
events as described above.
For the channel configuration investigated, no-slip boundary conditions are prescribed for the recity, where only $u(0)=-U$ and $u(2 h)=U$ are nonzero. Dirichlet boundary conditions $\Phi(0)=0$ and $\Phi(2 h)=$

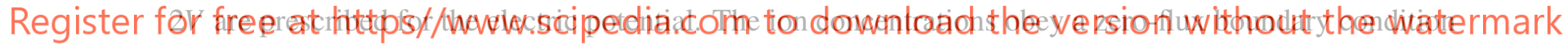

(right-hand side of (4) vanishes) so that the charges bare subject to redistribution and separation but the bulk charge is conserved. In this study, initially uniform concentrations, $c_{\perp}=c_{0}$, are prescribed for univalent ion species yielding zero bulk charge. The ODT model parameters used for this study, as detailed in section 2.2, are $C=10, Z=600$, and $\alpha=2 / 3$, which correspond to the planar Poiseuille flow parameters in [11].

Scaling of equations (1), (4) and (5) yields five independent dimensionless control parameters. These consist of the bulk Reynolds number, $R e=U h / \mathrm{v}$; the Schmidt number, $S c=\mathrm{v} / D$, of the ion species; the coupling constant, $\beta=\varepsilon V_{T}^{2} /(\rho v D)$, of electric forces; the dimensionless voltage, $\hat{V}=2 V / V_{T}$; and the normalized Debye length, $\hat{\lambda}_{\mathrm{D}}=\lambda_{\mathrm{D}} / h=\sqrt{\varepsilon V_{T} /\left(2 \rho c_{0} e h^{2}\right)}$, where $c_{0}=c_{ \pm, 0}$ denotes the uniform initial concentration of both ion species in addition to all other parameters reference values introduced above.

\subsection{Steady-state ion concentrations and electric field for the fluid at rest}

We begin with a validation of the electrokinetics module incorporated into the stand-alone ODT solver. This is done for the fluid at rest, but the electric variables are identical under laminar Couette flow. 

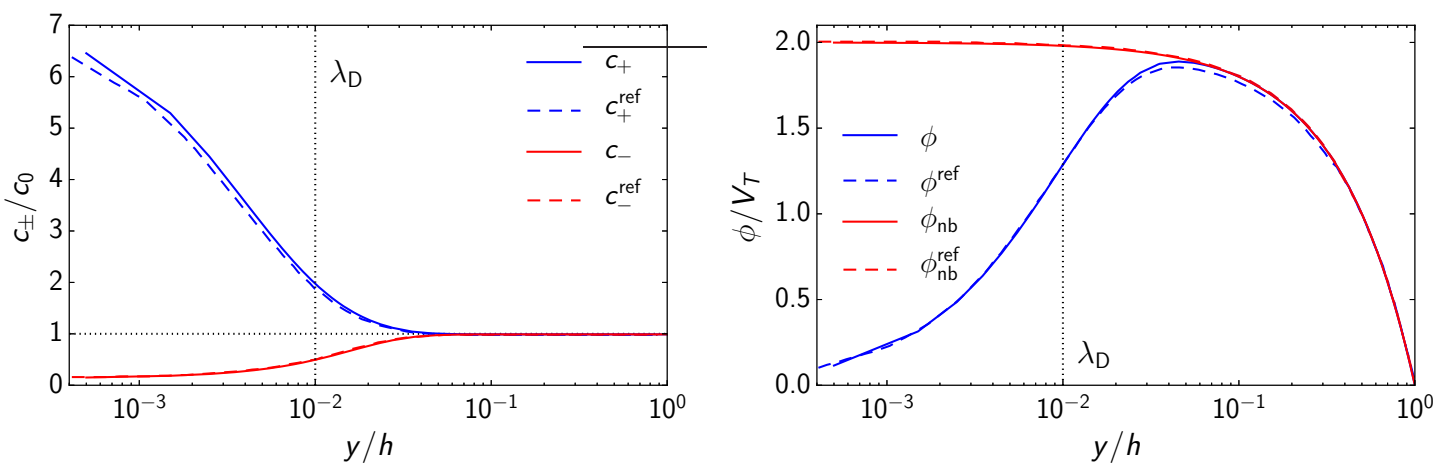

Figure 2: [left] Ion concentrations $c_{ \pm}$over the negative plate when the fluid is at rest for relevant control parameters $\hat{V}=4$, and $\hat{\lambda}_{\mathrm{D}}=\lambda_{\mathrm{D}} / h=0.01$ (vertical dotted line). Space charge due to the free charge-carrier density $\rho_{\mathrm{f}}=$ $e\left(c_{+}-c_{-}\right)$is confined in the Debye boundary layer. [right] Corresponding dimensionless perturbation electric field $\phi / V_{T}$ shields the boundary condition and reveals an effectively neutral bulk. Reference (superscript 'ref') data is from [6].

\section{Figure 2 shows steady-state ion concentrations and the corresponding perturbation potential over the lower (negative) plate in the case of quiescent flow due to the wall velocity $U=0$ at top and bottom. Concentrations $c_{ \pm}$and, hence, the free charge carrier density $\rho_{\mathrm{f}}=e\left(c_{+}-c_{-}\right)$, are localized in the vicin- ity of the wall in a thin exponential space-charge region that is denoted as Debye boundary layer with thickness $\lambda_{\mathrm{D}}=\sqrt{\varepsilon V_{T} /\left(2 \rho c_{0} e\right)}$. This layer effectively shields the boundary condition and yields neutral bulk as can be inferred from the perturbation poten
bulk with $\Phi=\phi+\phi_{0}=0$. The ODT results exhil
merical simulation (DNS) results [6]. The ion conce
data due to details of the spatial discretization and ne
depletes at the grounded bottom wall, which is reve} this set-up charge separation occurs at the wall due to interaction of drift and diffusion.

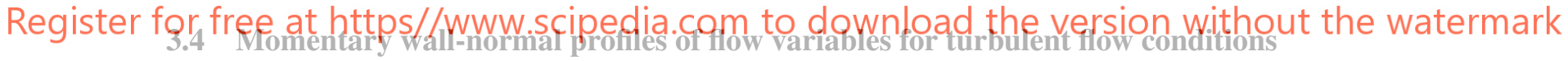

From here on we consider a turbulent flow coupled to EHD effects. For the present two-way (fully) coupled Couette flow configuration (figure 1), the flow is mechanically driven by a prescribed mean shear and exposed to an external electric field with electric potential $\phi_{0}(z)=2 V z$ due to electricly insulated top and bottom walls that are held at different voltages.

Figure 3 shows instantaneous wall-normal profiles of all flow variables that have been obtained by numerical solution of the ODT governing equations (1), (4) and (5). The profiles shown are 'wiggly' because ODT is not a statistical closure model in the classical sense but a stochastic turbulence model that resolves all relevant scales of the flow across the full channel height. These profiles fluctuate as time progresses so that conventional temporal and, if necessary, ensemble statistics can be performed for the ODT solution in order to assess the statistical flow properties. The difference in magnitude between two neighbouring extrema of a flow variable gives an impression of the magnitude of the fluctuations that are resolved within the lower-order ODT modeling framework. Note in this respect that the electric potential appears to be quite smooth along the $y$ coordinate, but it fluctuates quite strongly in time due to the limitations of the self-contained but one-dimensional Gauss law (5). This is addressed further in 

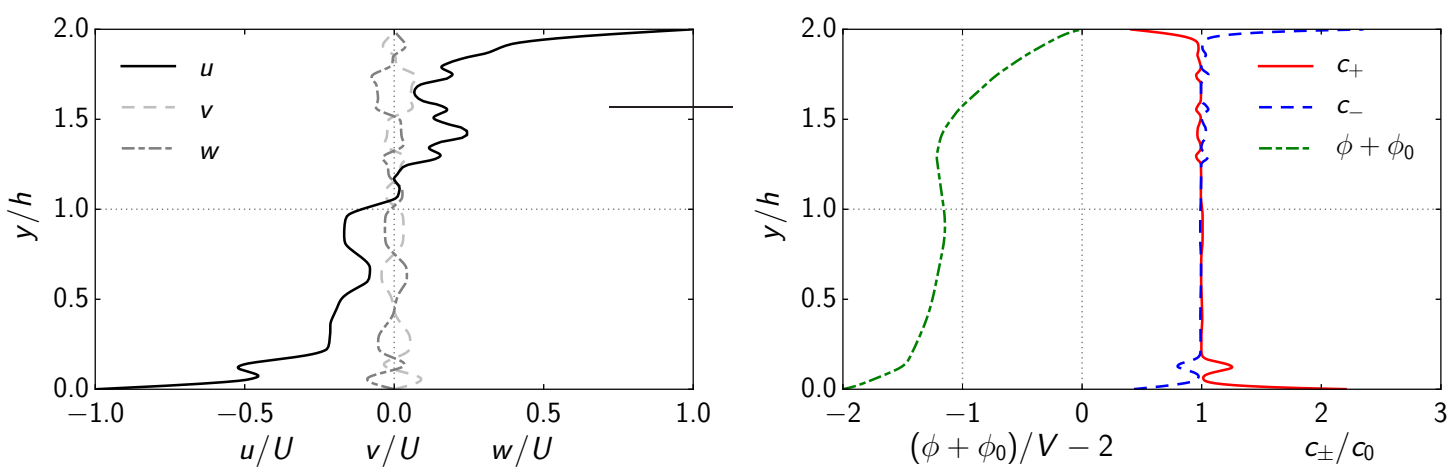

Figure 3: Instantaneous wall-normal profiles across the full channel of all dynamical variables normalized by bulk quantities for $R e=3000, S c=3, \hat{V}=10, \beta=1 / 2, \hat{\lambda}_{\mathrm{D}}=0.03$. [left] Cartesian components $u, v$, and $w$ of the velocity vector. [right] Total electric potential $\Phi=\phi+\phi_{0}$ (shifted for visualization) and ion concentrations $c_{ \pm}$.

section 4 below.

\subsection{EHD effects in the turbulent velocity and electric boundary layer}

Fluctuating property profiles of transient ODT simulations for $R e=3000$ and 12,000 have been averaged in time in order to investigate the role of EHD effects for the mean state of the turbulent boundary layer.

Figure 4 (left) therefore shows the dimensionless velocity deficit $u^{+}=|U-\bar{u}| / u_{\tau}$ over the boundary
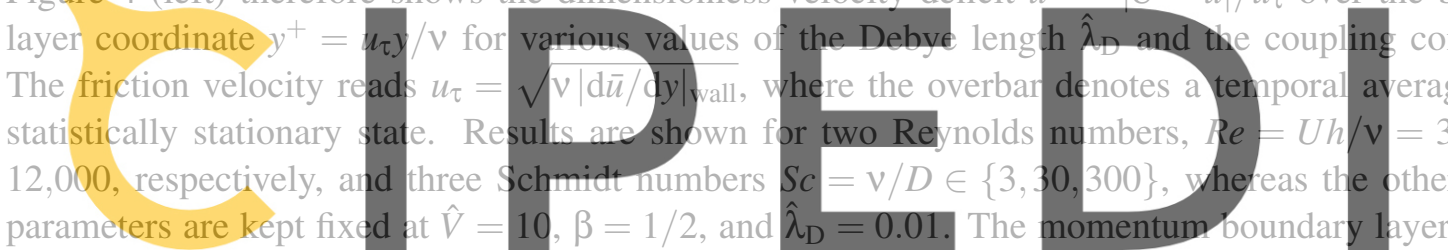

the viscous sublayer for $y^{+}<5$, the buffer layer for $5<y^{+}<30$, and the logarithmic layer for $y^{+}>30$.

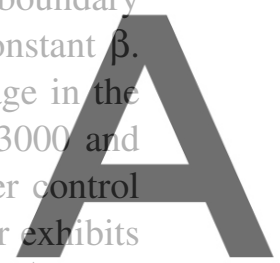

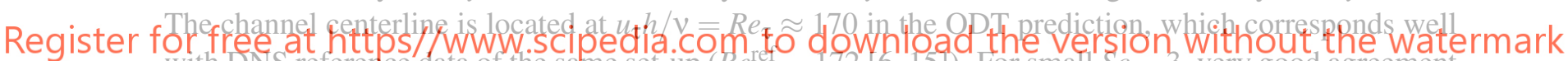
with DNS reference data of the same set-up $\left(R e_{\tau}^{\text {ret }}=172[6,15]\right)$. For small $S c=3$, very good agreement

is obtained between ODT and the empirical reference profile [16]. Here, as recently shown also in [10],

EHD effects yield a decrease of $\bar{u}^{+}$as can be seen for $y^{+} \gtrsim 100$ of the largest $R e=12,000$ and $S c=300$ investigated and hints at EHD-enhanced bulk turbulence.

Figure 4 (right) shows boundary layer profiles of the mean perturbation electric potential $\bar{\phi}$ corresponding to the velocity profiles in the left panel. For increasing $R e$ and $S c$ number, ODT predicts a reduction in magnitude of $\bar{\phi}$ and a shift of its maximum towards the bulk (larger $y^{+}$values). This implies less shielding of the electric boundary condition or, likewise, increased bulk electric fields under turbulent flow conditions.

It is worth to emphasize that turbulence, in general, enhances wall-normal transport processes irrespective of the dynamical coupling of the transported variables. Here, this means that we also need to consider the turbulent ion transport in more detail. The instantaneous flow profiles shown in figure 3 are consistent with the observation that the bulk is no longer neutral, but exhibits localized and fluctuating electric charges that originate from the near-wall Debye layer. The wall-normal transport associated with turbulent eddies (or eddy events) hence depletes the Debye layer by efficient redistribution of electric charge 

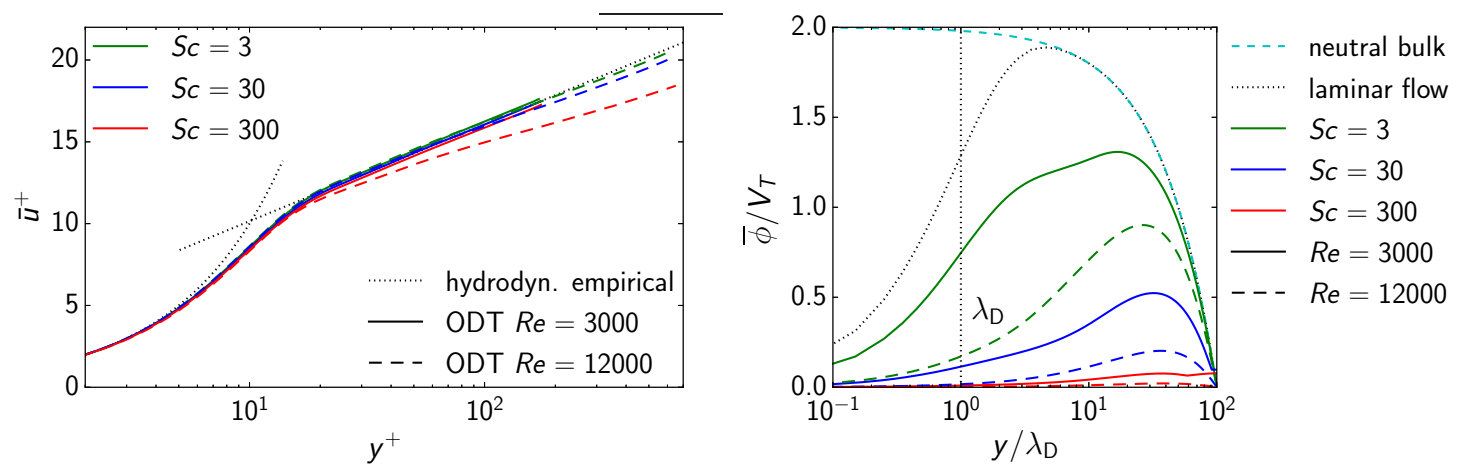

Figure 4: [left] Mean streamwise velocity deficit $\bar{u}^{+}$across the momentum boundary layer (coordinate $y^{+}$) for various $R e$ and $S c$ numbers but fixed $\hat{V}=4, \beta=1 / 2, \hat{\lambda}_{\mathrm{D}}=0.01$, and ODT model parameters. The empirical law-of-the-wall [16] applies for hydrodynamic flow $(\hat{V}=0)$ but is given for orientation. [right] Corresponding dimensionless mean perturbation electric potential $\bar{\phi} / V_{T}$ over a differently scaled abscissa. The Debye length scale $\lambda_{\mathrm{D}}$ is given as vertical dotted line. Note that $\phi$ for laminar Couette flow is identical to the fluid at rest.

carriers across the turbulent boundary layer into the bulk of fluid, where the ion species are finally mixed. This mechanism is closely tied to the fact that the electric charge separation is limited by slow molecular drift and diffusion currents and localized to the electricly insulated (zero-flux) wall used here.

Dimensional analysis of the ion species equation (4) reveals that turbulent charge carrier (ion) advection, which is here modeled by the stocmastic term $\mathcal{E}_{ \pm}$, is of the order $O(P e=S c R e)$ - Jarger than molecular drift and diffusion currents. increasing Re and $S c$ nu A similar conclusion with on electric forces in th these effects manifest themselve by a reduction
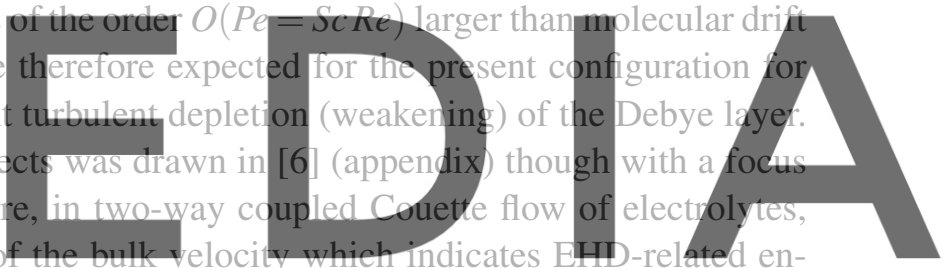
hancement of the turbulent drag.

Register for free at https//www.scipedia.com to download the version without the watermark 4 ONE-WAY COUPLING: EHD-EIFECTS IN TURBULENT PLANAR POISEUILLE FLOW

Despite the simpier dynamics addressed by the one-way coupling formulation, the study presented here is nonetheless a complex case due to our intended use of stationary 2-D electric fields, which agree with those of a wire-plate electrostatic precipitator.

\subsection{Form of the Poisson-Nernst-Planck equation and of Gauss's law}

The static electric field distribution used in the one-way coupled Poiseuille case is calculated in a fully two-dimensional framework. The Poisson-Nernst-Planck equation for ion charge density neglects in this case ionic diffusion in comparison to equation (4). Transient effects due to fluctuations of the perturbation electric potential are also neglected by taking the limit of weak charge carrier densities and high Schmidt numbers, which is here more appropriately expressed as the limit of a small mobility ratio $M_{\mu}=U_{\mathrm{b}} H /\left(V \mu_{\text {ion }}\right) \ll 1$, where $U_{\mathrm{b}}$ is a (domain averaged) bulk velocity, and $\mu_{\text {ion }}=D_{\text {ion }} / V_{T}$ is the electric mobility, specialized in this case for positive ions in air. The resulting Poisson-Nernst-Planck equation has purely ionic drift that originates from a corresponding electric drift current density vector 

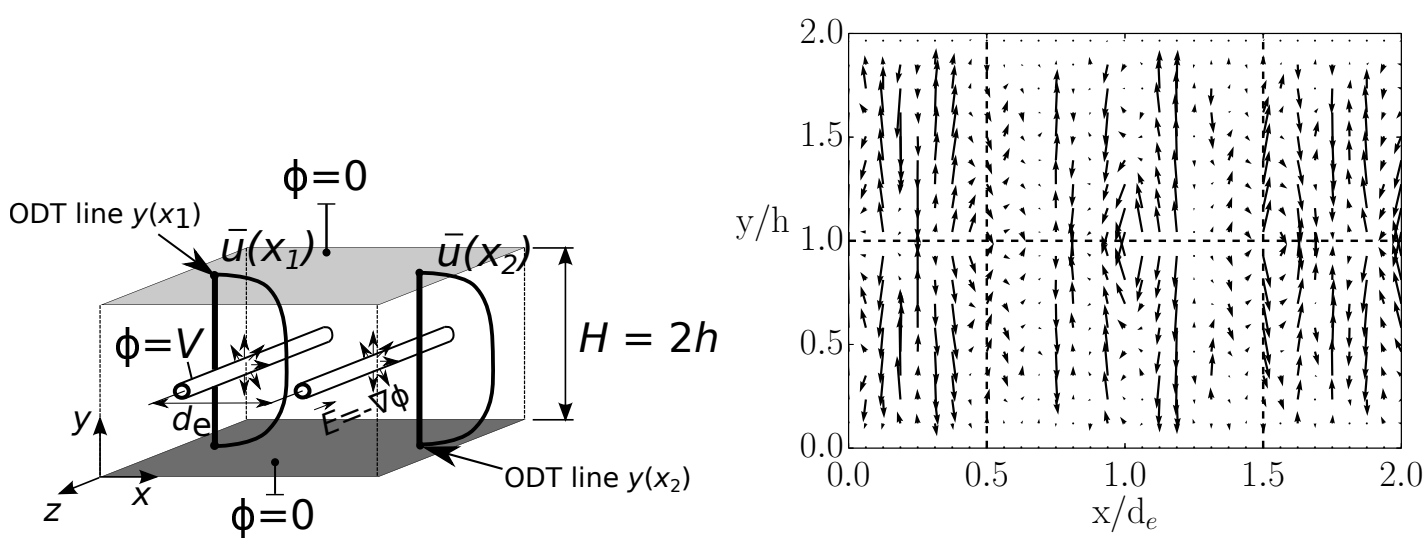

Figure 5: [left] Sketch of the EHD Poiseuille flow configuration. The streamwise advected Lagrangian ODT domain is denoted as 'ODT line' and is visualized at two different streamwise positions, $x_{1}$ and $x_{2}$. A graphical representation of the radially divergent electric field $\vec{E}=-\vec{\nabla} \Phi$ originating from the (point) wire electrodes is shown. [right] Ensemble average of the velocity field between two electrodes in the simulated EHD Poiseuille flow for the high voltage case $V_{\mathrm{B}}$. The distribution corresponds to the secondary flow, that is, $\vec{u}^{\prime}(x, y)=\langle\vec{u}\rangle(x, y)-\left(U_{\mathrm{b}}, 0,0\right)^{T}$, where $U_{\mathrm{b}}$ is the domain and streamwise-averaged bulk velocity, and $\langle\cdot\rangle$ a conventional ensemble average. This image can be compared to the fluctuating velocity field presented by [5].

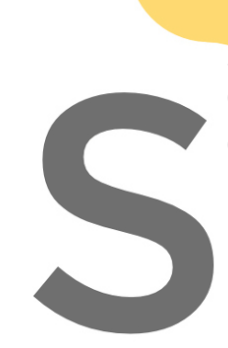

$-\rho_{\mathrm{f}} \mu_{\mathrm{ion}} \vec{\nabla} \Phi$ equations (4) and (5), p electric permittivity. Ther
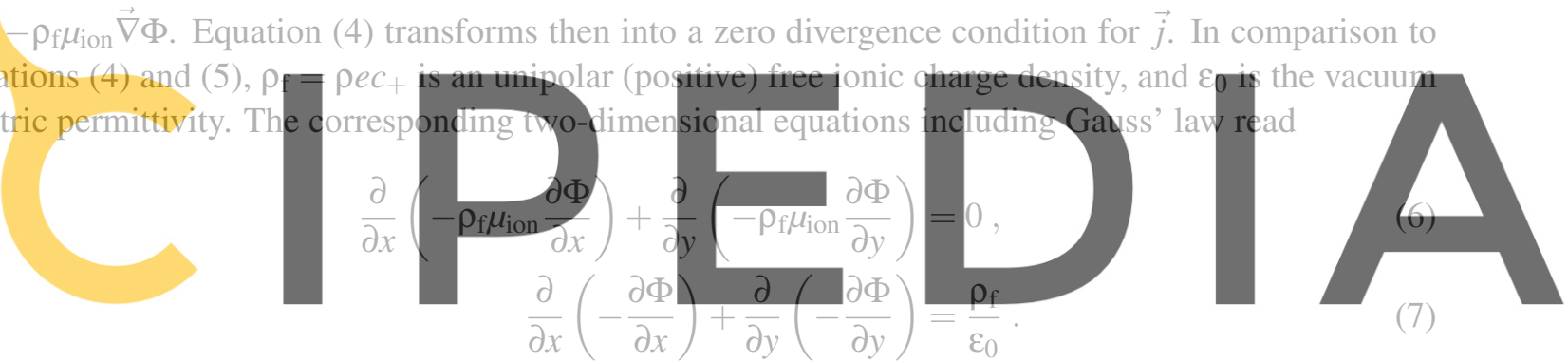

Register for free at https//www.scipedia.com to download the version without the watermark 4.2 Flow configuration, simulation strategy and control parameters

Figure 5 shows a sketch of the one-way coupled flow configuration investigated. The ODT domain ('ODT line') for self-contained flow simulations is aligned with the vertical or wall-normal (y) direction and spans the entire height of the channel $H=2 h=0.04 \mathrm{~m}$. The lateral direction $(z)$ is taken as infinity, and an electric current per unit width $I_{\text {plate }} / W$ is considered as a boundary condition at the plates. Wire electrodes aligned in the lateral direction $z$ are arranged in a periodic configuration with a given streamwise inter-electrode separation $d_{\mathrm{e}}=0.0628 \mathrm{~m}$. The infinite periodic electrode arrangement, as well as the assumption of a constant property flow, induce statistical homogeneity in direction $x$. For the channel configuration investigated, no-slip boundary conditions are prescribed for the velocity, $u(0)=0$ and $u(2 h)=0$. Dirichlet boundary conditions for the electric potential $\Phi=V$ are prescribed at the wire electrodes, as well as at the channel walls, $\Phi=0$.

In order to solve equations (6)-(7), an iterative scheme is used, where the value of the charge density at the electrode is iterated and used in order to calculate the 2-D charge density field by (6) and a corresponding 2-D electric potential field by (7). The iteration takes place by comparing the resulting electric current per unit width at the plate against the input boundary condition $I_{\text {plate }} / W$, see $[9,17]$ for details. 
The generated 2-D electric current density field from equations (6)-(7) is supplied as background field to S-ODT simulations. That is, S-ODT simulations are only concerned with the solution for momentum conservation, or conversely, with the integration of the velocity PDE (2) in a streamwise-marching scheme, analogous to the time integration of (1) in the two-way coupled case. As explained in 2.2, we do not consider any transfer of electric potential energy into kinetic energy during stochastic eddy events. Any source or sink of kinetic energy in this sense, is counted in the flow forcing by means of a fixed pressure gradient (FPG), as written in equation (2). Due to this reason, a decomposition of the electric current density vector is motivated following a Helmholtz decomposition. Decomposing $\vec{j}$ into an irrotational $\vec{j}_{I}=-\vec{\nabla}\left(\rho_{\mathrm{f}} \mu_{\text {ion }} \Phi\right)$ and a solenoidal component $\vec{j}_{S}=\mu_{\text {ion }} \Phi \vec{\nabla} \rho_{\mathrm{f}}$, such that $\vec{j}=\vec{j}_{S}+\vec{j}_{I}$, allows us to neglect the irrotational part (kinetic energy source or sink), and incorporate into the model formulation the sole effects of $\vec{j}_{S}$ [9]. The latter are the only effects on turbulence or eddy event probabilities by the electric current density field. Having said that, $\vec{e}_{x} \cdot \vec{j}_{S} / \mu_{\text {ion }}$ is incorporated in the deterministic streamwise advancement as an additional body force in (2), while $\vec{e}_{y} \cdot \vec{j}_{S} / \mu_{\text {ion }}$ is incorporated in the eddy event probability calculation by equation (3). This is the way to formally incorporate the 2-D electric fields into the one-dimensional turbulence framework (S-ODT).

The scaling of equation (2), the only relevant dynamical equation for the one-way coupled case, yields two independent dimensionless control parameters. These are the bulk Reynolds number, $R e=U_{\mathrm{b}} h / \mathrm{v}$ and the electrohydrodynamic number, $N_{\mathrm{EHD}}=\sqrt{(I / W)\left[H^{2} /\left(4 \mu_{\mathrm{ion}} \rho v^{2}\right)\right]}$. Alternatively, Re can be expressed in terms of the skin friction coefficient $C_{\mathrm{f}}=2 u_{\tau}^{2} / U_{\mathrm{b}}^{2}$ and $R e_{\tau}=u_{\tau} h / \mathrm{v}$. Recall that the friction velocity $u_{\tau}$ is imposed in this case by the FPG. In the simulations, a value $R e_{\tau}=108$ is imposed. Two

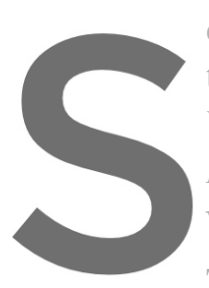
different electrohydrody

the reference case at zero $V_{A}=32 \times 10^{3} \mathrm{~V}$ and $\mathrm{V}$ $I_{\text {plate }, \mathrm{A} / W} / \mathrm{W} \times 10^{-4} \mathrm{~A}$ $\mathrm{V}=1.66 \times 10^{-5} \mathrm{~m}^{2} / \mathrm{s}$, and the ionic mobility $\mu_{\text {ion }}$ The ODT model parameters used for this study,
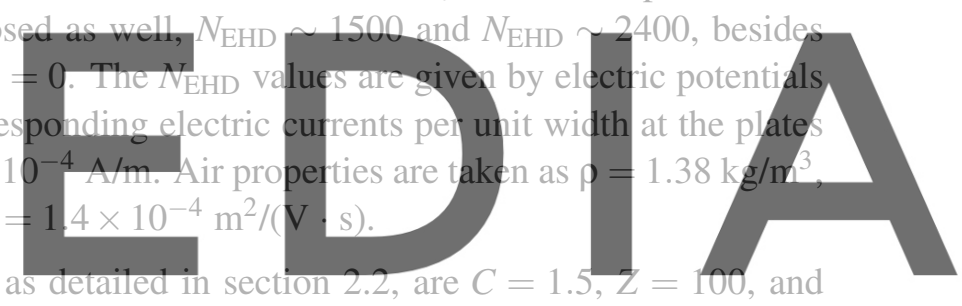
$\alpha=0$, such that only the streamwise velocity component is solved by the formulation. The crosswise

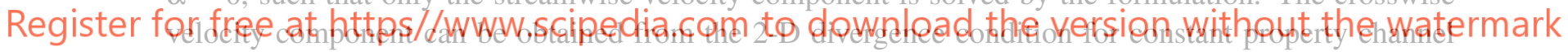
flow. It is noted that we consider a scaling of the model parameter $C$ with respect to $N_{\text {EHD }}$ (see [9]).

\subsection{EHD effects on the mean Poiseuille flow statistics}

Figure 5[right] shows the 2-D fluctuating velocity field obtained with S-ODT simulations in the high voltage case. It is seen that streamwise variations of the streamwise velocity are negligible in comparison to the variations of the crosswise velocity. Interestingly enough, S-ODT is still able to get some of the vortical structures expected for this type of flow, see [5].

Figure 6[left] shows the statistically homogeneous mean velocity profile in wall-normal coordinates. Unlike in the two-way coupling case, the flow forcing done by a FPG results in an increase of the bulk velocity with increasing magnitude of $N_{\mathrm{EHD}}$. That is, a drag reduction effect is noticed. Figure 6[right] shows the viscous and turbulent shear stress in wall-normal coordinates. The increased magnitude of the electric body force results in an overall decrease of the Reynolds stress, and an increase of the viscous stress, that is, an apparent sign of relaminarization of the flow, or at least, of a reduction of turbulence, due to its subordination to the increased magnitude of the Coulomb force. 

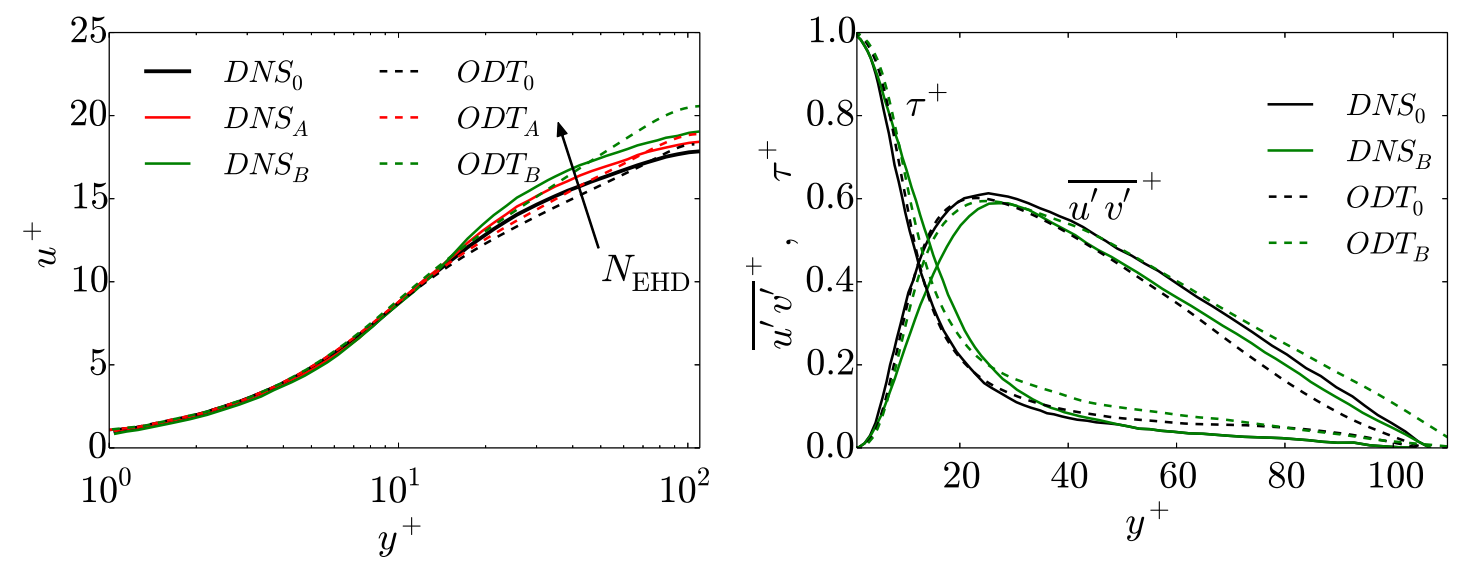

Figure 6: [left] Mean streamwise velocity profile for the reference, low and high $N_{\text {EHD }}$ numbers. [right] Corresponding normalized viscous and Reynolds stress for the reference and high $N_{\mathrm{EHD}}$ cases. Statistics have been obtained by Reynolds averaging under the assumption of streamwise homogeneity at infinite domain length. The mean velocity profile is normalized by $u_{\tau}$ and the mass-specific stresses by $u_{\tau}^{2}$. DNS reference data are from [5].

\section{CONCLUDING REMARKS}

Stochastic ODT simulations of EHD-enhanced turbulent flows have been conducted and compared to available reference data. A one-way and a two-way coupling strategy have been proposed and tested. Both strategies yield self-contained flow simulations that utilize ODT as a lower-order stand-alone tool. The approach makes investigation of broad control parameter ranges feasible which is currently beyond the capabilities of DNS, and very challenging even for large-eddy simulations (LESs). The main reason for this roots in the interactions between inertial, viscous, and Coulomb forces, as well as turbulentadvective and drift-diffusion currents of the charge carriers (ion species) down to the microscales of the flow. In the hydrodynamic flow regime with moderately mobile ions, ODT is able to reproduce relevant low-order velocity statistics. The model furthermore predicts that EHD-turbulence interactions in wallbounded flow will occur only for large enough $R e$ and $S c$ numbers, but they will manifest themselves dependent on the application. In two-way coupled EHD Couette flow of electrolytes turbulence intensification and mean velocity reduction, that is, turbulent drag increase, are predicted by ODT. By contrast, for the one-way coupled planar EHD Poiseuille fow in an electrostatic precipitator, localization of bulk turbulence together with drag decrease have been obtained. While the underlying physical processes are not fully understood yet, these results indicate robustness of the physics-based modeling strategy. This seems to overcompensate some shortcomings of the one-dimensional formulation as seen for Gauss' law.

\section{Acknowledgements}

We thank David O. Lignell for sharing his adaptive ODT implementation [18].

This work was supported by the European Regional Development Fund (EFRE), grant no. STaF 23035000.

\section{REFERENCES}

[1] Ohadi, M. M., Nelson, D. A. and Zia, S. Heat transfer enhancement of laminar and turbulent pipe flow via corona discharge. Int. J. Heat Mass Transfer (1991) 34(4-5):1175-1187. 
[2] Nelson, D. A., Zia, S., Whipple, R. L. and Ohadi, M. M. Corona discharge effects on heat transfer and pressure drop in tube flows. J. Enh. Heat Transfer (2000) 7:81-95.

[3] Cha, M. S., Lee, S. M., Kim, K. T. and Chung, S. H. Soot suppression by nonthermal plasma in coflow jet diffusion flames using a dielectric barrier discharge. Combust. Flame (2005) 141:438447.

[4] Yamamoto, T. and Velkoff, H. R. Electrohydrodynamics in an electrostatic precipitator. J. Fluid Mech. (1981) 108:1-18.

[5] Soldati, A. and Banerjee, S. Turbulence modification by large-scale organized electrohydrodynamic flows. Phys. Fluids (1998) 10(7):1742.

[6] Ostilla-Mónico, R. and Lee, A. A. Controlling turbulent drag across electrolytes using electric fields. Faraday Discuss. (2017) 199:159-173.

[7] Kerstein, A. R. One-dimensional turbulence: model formulation and application to homogeneous turbulence, shear flows, and buoyant stratified flows. J. Fluid Mech. (1999) 392:277-334.

[8] Kerstein, A. R., Ashurst, W. T., Wunsch, S. and Nilsen, V. One-dimensional turbulence: vector formulation and application to free shear flows. J. Fluid Mech. (2001) 447:85-109.

[9] Medina Mendez, J. A. Application of the One-Dimensional Turbulence model to electrohydrodynamically enhanced internally forced convective flows. $\mathrm{PhD}$ Thesis. Faculty 3, Brandenburg University of Technology Cottbus-Senftenberg, Germany (2020).

[10] Klein, M. and Schmidt, H. Towards a stochastic model for electrohydrodynamic turbulence with application to electrolytes. Proc. Appl. Math. Mech. (2020) 20:e202000128.

[11] Lignell, D. O., Kerstein, A. R., Sun, G. and Monson, E. I. Mesh adaption for efficient multiscale implementation of one-dimensional turbulence. Theor. Comp. Fluid Dyn. (2013) 27(3):273-295.

[12] Wunsch, S. and Kerstein, A. R. A stochastic model for high-Rayleigh-number convection. J. Fluid Mech. (2005) 528:173-205.

[13] Gonzalez-Juez, E. D., Kerstein, A. R. and Lignell, D. O. Fluxes across double-diffusive interfaces: a one-dimensional-turbulence study. J. Fluid Mech. (2011) 677:218-254.

[14] Jozefik, Z., Kerstein, A. R., Schmidt, H., Sgouria L., Kolla, H., Chen, J. H., One-dimensional turbulence modeling of a turbulent counterflow flame with comparison to DNS Combust. Flame (2015) 162:2999-3015.

[15] Pirozzoli, S., Bernardini, M. and Orlandi, P. Turbulence statistics in Couette flow at high Reynolds number. J. Fluid Mech. (2014) 758:327-343.

[16] Marusic, I., McKeon, B. J., Monkewitz, P. A., Nagib, H. N., Smits, A. J. and Sreenivasan, K. R. Wall-bounded turbulent flows at high Reynolds numbers: Recent advances and key issues. Phys. Fluids (2010) 22:065103.

[17] Yamamoto, T. Electrohydrodynamic secondary flow interaction in an electrostatic precipitator. $\mathrm{PhD}$ Thesis. The Ohio State University, Ohio, U.S.A. (1979).

[18] Stephens, V. B. and Lignell, D. O. Computationally efficient modeling and simulation of turbulent flows. SoftwareX (2021) 13:100641. 